\title{
Pulsed Plasma Surface Modifications (PPSM) from an Industrial Perspective. Pico-Technology, Nano-Structures and Novel Structures with Unique Properties
}

\author{
Rune Harald Hoel
}

MOTecH Plasma a.s, PB 540, NO-1411 Kolbotn, Norway

Non-equilibrium, non-thermal vacuum-plasmas provide the possibility to do high-temperature materials physics at low temperatures. Through ionization, stable, non-reactive gases may be converted into highly reactive species which may penetrate into surfaces, diffuse into the base material and convert the core structures into novel, metastable structures with unique properties.

Various steels and nickel-based alloys have been surface modified in vacuum (30-300Pa) at elevated temperatures and their structure and properties have been characterized. In such plasmas, nitrogen-, carbon-. and oxygen-rich gases were ionized by connecting a directly pulsed, negative voltage of $450-600 \mathrm{~V}$ to the work piece. Under such conditions, stable, non-reactive gas molecules like $\mathrm{N}_{2}$ will split into a mixture of more reactive atomic species and very reactive positive ions (e.g. $\mathrm{N}^{+}$). An important feature of the ionization process is that the diameter of an atom may be reduced from 72 picometers to the 16 picometers for an ion. Hence, by manipulating species in the picometer range and applying a negative voltage to the crystal lattice, it is possible to force the positive ions to penetrate into a lattice at relatively low temperatures $\left(<400 \mathrm{C}^{\circ}\right)$, as illustrated in Fig. 1 . As the ions enter the crystal they will diffuse into the lattice and either remain in solid solution, form clusters, or react with nitride formers to form precipitates. The nitrogen may also react with the main constituent of the alloy and form a continuous, hard surface layer.

Fig. 2 shows how a hard, continuous, heavily twinned surface layer may form on superduplex steels. Such a layer consists of a novel, metastable structure, often termed an extended austenite phase, a gamma-phase, or an S-phase. In low alloy steels, compound layers with $\mathrm{Fe}_{2-3} \mathrm{~N}$ and/or $\mathrm{Fe}_{4} \mathrm{~N}$ structures are formed. Directly underneath these hard surface layers is a so-called diffusion zone, which may contain nitride precipitates, clusters and/or nitrogen in solid solution. The diffusion zone consists of a graded structure with a gradual transition from the soft, unaffected core material to the hard, wear- and corrosion protective layer. The hardness profile in Fig. 3 confirms such a graded structure and property (AISI 4140). The diffusion zones thus provide an important mechanical support for the hard, top surface layers, in order to avoid the so-called eggshell effect. Different types of S-phases may be produced, depending on the gas-mixtures. In a nitrogen rich plasma the $\mathrm{S}_{\mathrm{N}}$-phase

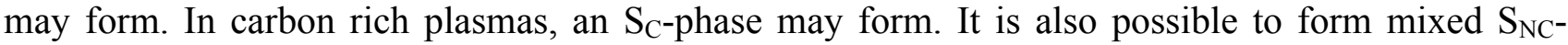
phases. The different S-phases possess different properties, and the optimum property is often achieved by dividing the S-layer into different sub-layers, as depicted in Fig. 4. Tibological and corrosion properties may also be optimized through post-oxidation (Fig. 4). Such structures may also protect against hydrogen embrittlement [1]. Through the manipulation of gas mixtures and the temperature, it is also possible to tailor structures with or without precipitates. Figs. 5 and 6 show nano-sized precipitates intentionally grown within the extended austenite phase. EPMA also shows that nitrogen may reach colossal supersaturation levels of up to $30 \%$. Similar effects have been observed for carbon by Ernst et al [2]. Such structures result in very high residual, compressive stresses which are beneficial against fatigue.

Unique structures and combinations of various properties may be achieved through pulsed plasma surface modifications. However, there is a significant need for further microstructural characterizations both of the extended austenite phases, clusters and precipitates. Precipitate density 
and Orowan hardening levels should be determined and single crystal phase boundaries should be characterized.

\section{References:}

[1] M. Asgari et al, Corrosion Science 62 (2012), p. 51.

[2] F. Ernst et al, Acta Materialia 59 (2011), p. 2268.

[3] The electron microscopy collaboration with the Max Planck Institute, Stuttgart (Dr. E. Bischoff) and the NTNU University of Trondheim (Dr. R Giri) are greatly appreciated.
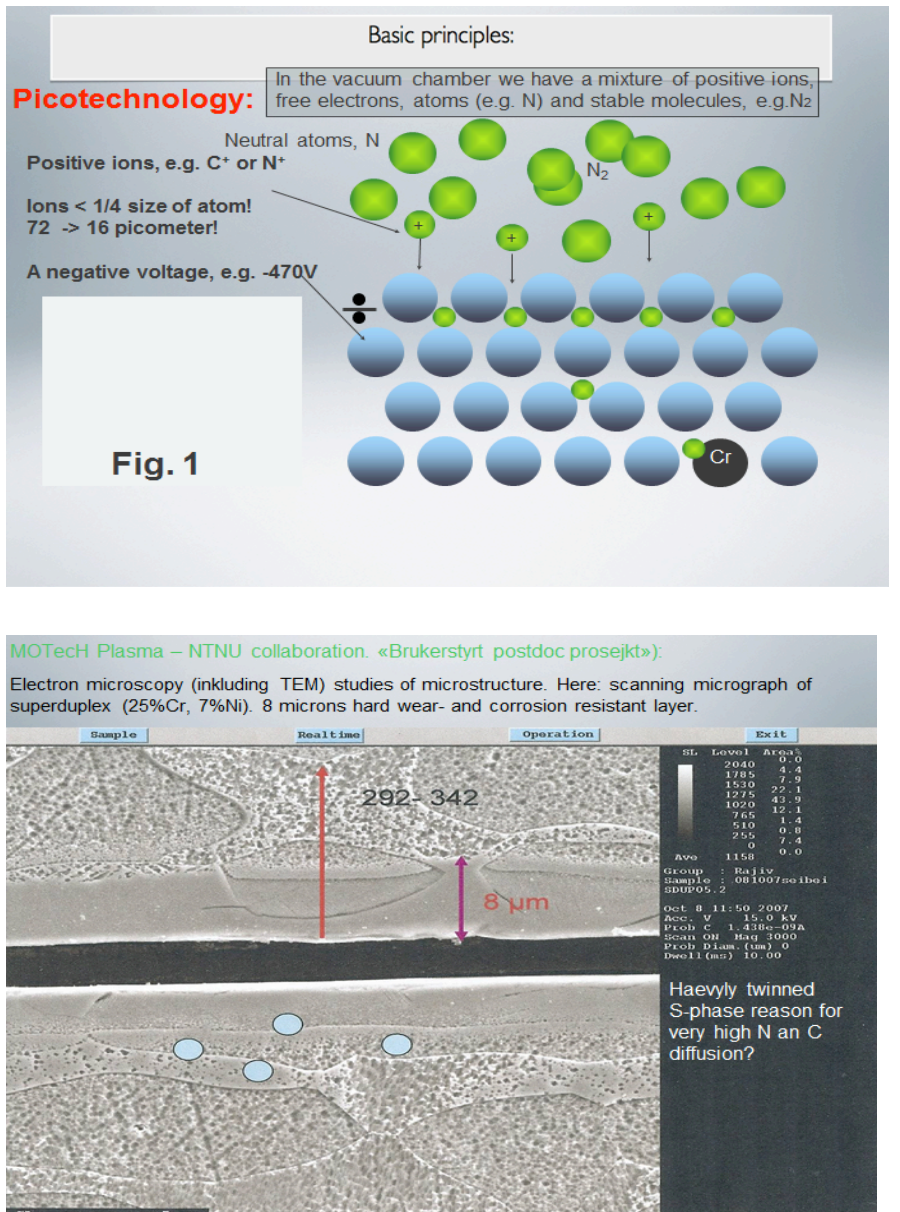

Fig. 2, $8 \mu \mathrm{m} \mathrm{S-/gamma-phase} \mathrm{on} \mathrm{superduplex}$

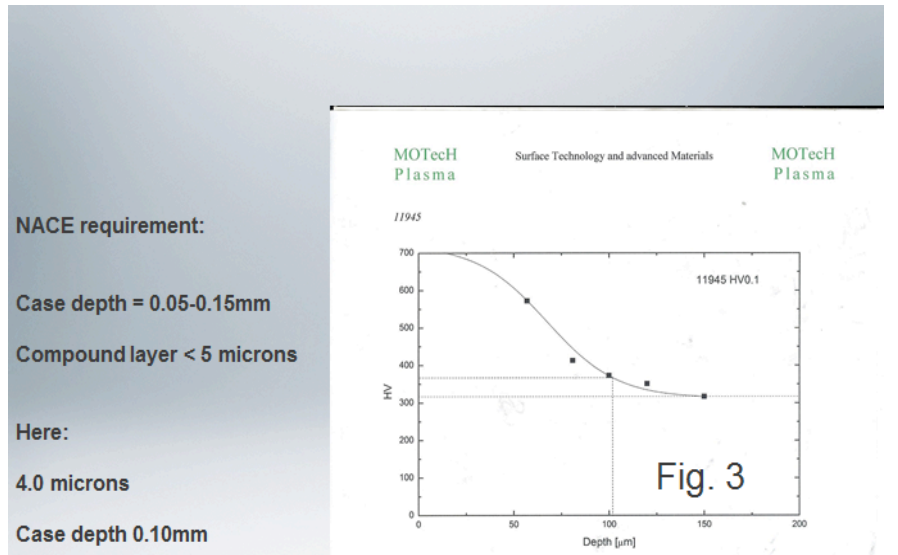

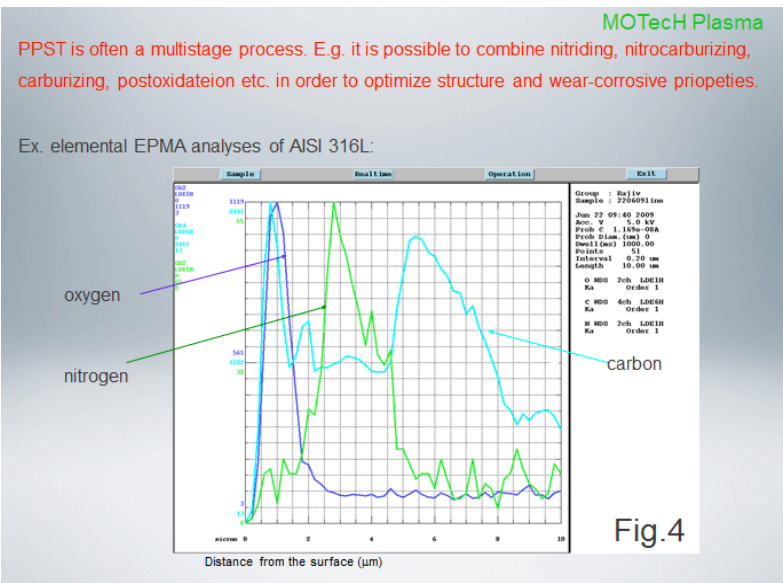

Cross-section (SEM and TEM): $20 \mathrm{~nm}$ particles (dark field). Complex S-phase structures.

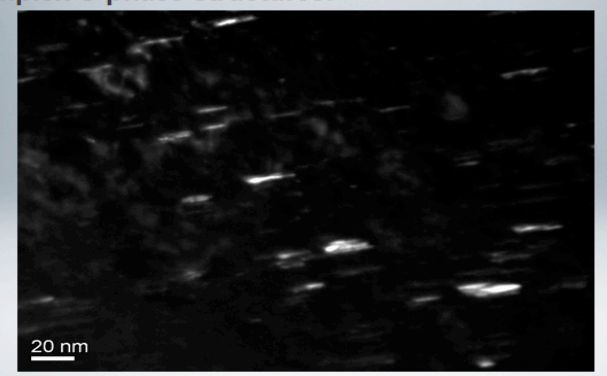

Fig. 5

XRD used to confirm presence of gamma phases and presence/absence

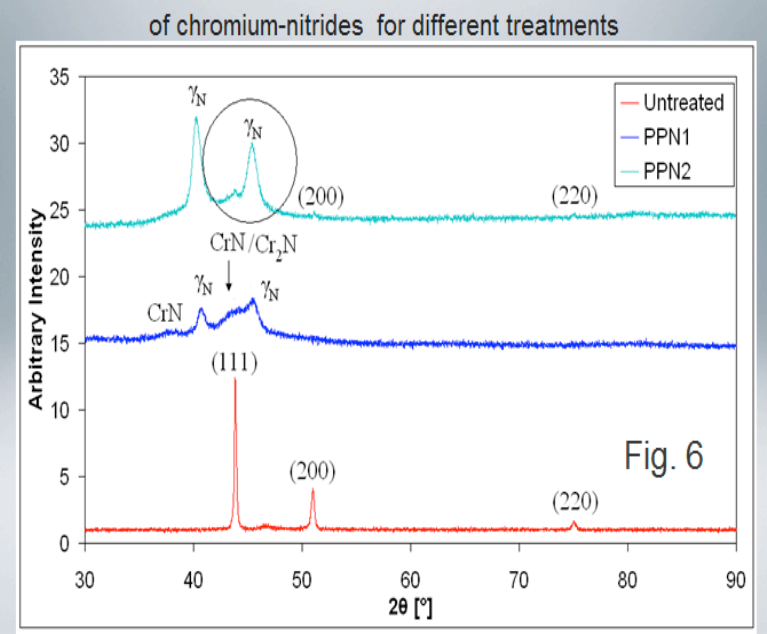

